\title{
Resolving Internal Magnetic Structures of Skyrmions by Lorentz Electron Ptychography
}

Zhen Chen $^{1}$, Emrah Turgut ${ }^{2}$, Yi Jiang ${ }^{3}$, Kayla X. Nguyen ${ }^{4}$, Gabriela C. Correa ${ }^{5}$, Matthew J. Stolt ${ }^{6}$, Song Jin $^{6}$, Daniel C. Ralph ${ }^{7}$, Gregory D. Fuchs ${ }^{1}$ and David A. Muller ${ }^{1,8^{*}}$

${ }^{1 .}$ School of Applied and Engineering Physics, Cornell University, Ithaca, NY 14853, USA.

2. Department of Physics, Oklahoma State University, Stillwater, OK 74078, USA.

3. Advanced Photon Source, Argonne National Laboratory, Lemont, IL 60439, USA.

4. Department of Chemistry and Chemical Biology, Cornell University, Ithaca, NY 14853, USA.

5. Department of Materials Science and Engineering, Cornell University, Ithaca, NY 14853, USA.

6. Department of Chemistry, University of Wisconsin-Madison, Madison, WI 53706, USA.

7. Department of Physics, Cornell University, Ithaca, NY 14853, USA.

${ }^{8 .}$ Kavli Institute at Cornell for Nanoscale Science, Ithaca, NY 14853, USA.

* Corresponding author: david.a.muller@cornell.edu

Due to topological protection, magnetic spin textures such as skyrmions can be stabilized and manipulated even at nanometer scale [1]. Observing such small skyrmions, and especially their internal spin structures, requires high resolution magnetic imaging techniques. Using differential phase contrast (DPC), Lorentz STEM can directly measure the magnetic induction field via the deflection of electron beam from Lorentz force [2]. However, the field-free condition required for preserving the intrinsic magnetic structures limits the spatial resolution to a few nanometers. The measurement precision of the beam deflection is inversely proportional to the square root of the total number of electrons in the incident beam [2], and therefore, until the recent development of high-dynamic range detectors, the performance of traditional DPC has been poor compared to Lorentz TEM or holography.

Here, by using a new electron microscope pixel array detector (EMPAD) with a dynamic range up to $1,000,000$ electrons / pixel and better than single electron sensitivity [3], we achieved a measurement precision better than $0.1 \mu \mathrm{rad}$ of beam deflection in Lorentz STEM mode. This allows high-quality planview imaging of magnetic domain structures in cobalt films with only 3-5 atomic layers, even when buried between thick Pt and Ta layers needed to stabilize the interfacial spin couplings. Figure 1 (a) shows the 4D Lorentz STEM setup. As the main probe-forming lens in Lorentz STEM is replaced by a condenser lens with a much larger spherical aberration, the probe size is limited to $3 \sim 4 \mathrm{~nm}$. To further increase the resolution and reduce the image distortions due to the residual aberrations and partial coherence in the probe, we adapted mixed-state ptychography which was initially proposed for compensating for the decoherence of X-ray imaging systems [4]. Using mixed-state ptychography in Lorentz mode, i.e., Lorentz electron ptychography, we reconstructed the phase change of the electron wave function induced by the magnetic field of a skyrmion lattice structure in a single crystal FeGe, as shown in figure 1 (b). The magnetic induction field calculated by differentiating the phase is shown in figure 1 (c) with the grayscale showing the magnitude and the color showing the direction. Figure 1 (d) shows the vector plot of the magnetic induction field. Magnetization discontinuities around the skyrmions are illustrated by the sharp hexagonal edges in the direction map shown in figure 1 (d). Furthermore, an anti-vortex at the skyrmion unit-cell boundary is experimentally observed, as shown in figure 1 (e). These internal spin structures and their singular points are crucial for accurate description of the topological properties and complete understanding of the dynamics of magnetic skyrmions. 
Figure 2 shows a quantitative comparison of the magnetic induction field maps determined from Lorentz electron ptychography and direct deflection of the beam (center-of-mass, CoM). Electron ptychography utilizes the entire diffraction pattern and accounts for the probe distortions due to residual aberrations and partial coherence (figure $2(\mathrm{~g})$ ). Therefore, it further improves the signal-to-noise and precision of the induction field map compared with direct CoM. As shown in figure 2 (e) \& (f), electron ptychography reduces the uncertainty of the field vectors especially near the magnetic singularity points such as at the corner of skyrmion lattice. Moreover, electron ptychography corrects for the damping from the electron probe shape and asymmetries, leading to more accurate measurements of the magnetic field, which is illustrated as the enhancement of the field magnitude in figure $2(\mathrm{~h})$.

References:

[1] R Wiesendanger, Rev. Mod. Phys. 81 (2009), p. 1495.

[2] J Chapman et al., Ultramicroscopy 3 (1978), p. 203.

[3] MW Tate et al., Microsc. Microanal. 22 (2016), p. 237.

[4] P Thibault and A Menzel, Nature 494 (2013), p. 68.

[5] Research supported by DARPA (D18AC00009) \& NSF (DMR-1539918, DMR-1719875).
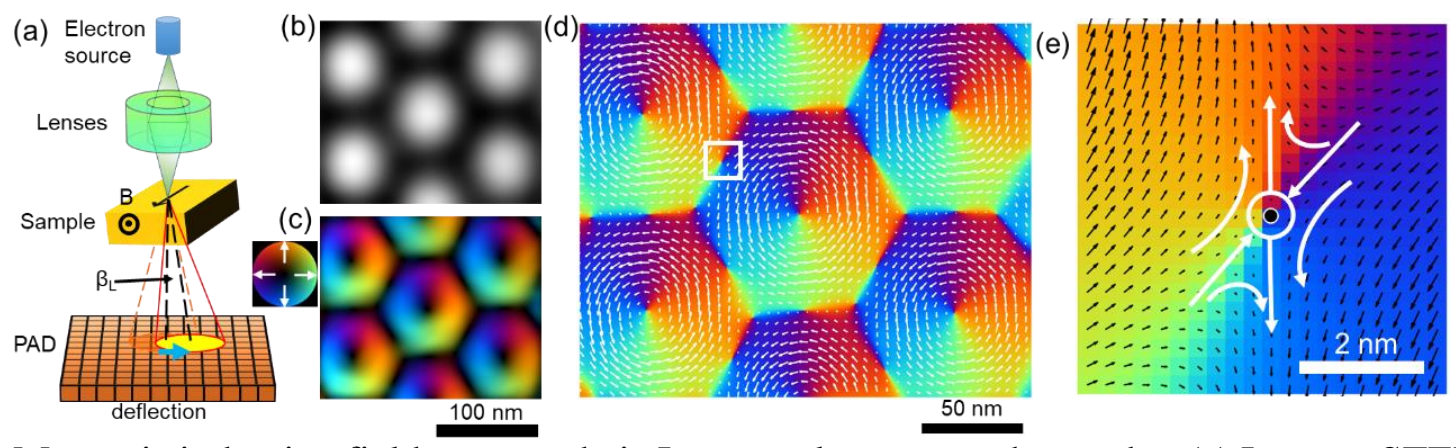

Figure 1. Magnetic induction field measured via Lorentz electron ptychography. (a) Lorentz STEM setup. (b) Phase shift reconstructed via Lorentz electron ptychography for skyrmion lattice in single crystal FeGe at 94 K. (c) Magnetic induction field vector calculated from the phase shift. (d) Magnetic induction field direction with arrows representing the field vectors. (e) An anti-vortex from the region marked on (d).

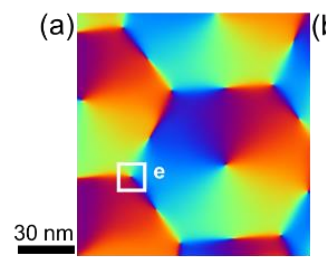

(e)

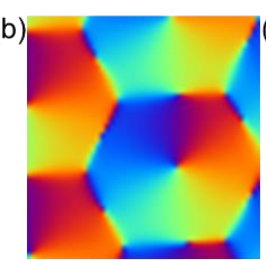

(f)

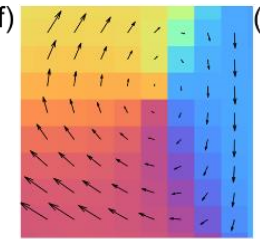

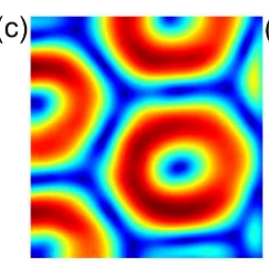

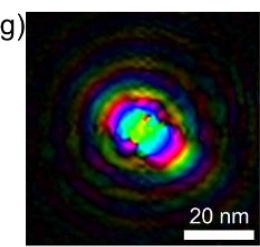

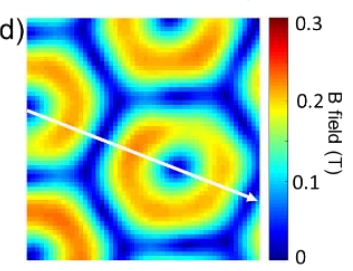

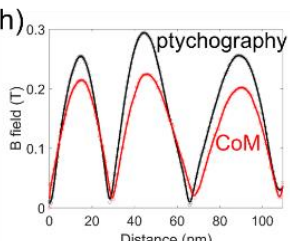

Figure 2. Comparison of magnetic induction field determined from ptychography and direct center of mass (CoM). (a) \& (c) Field direction and magnitude from ptychography; (b) \& (d) Field direction and magnitude from CoM; (e) \& (f) Field vectors (selected regions marked on (a)) determined from ptychography (e) and CoM (f); (g) Electron probe retrieved via ptychography; (h) Line profiles of field magnitude from ptychography (black) and CoM (red) (profile of white line in (d)). 